\title{
OPTIMAL SELECTION OF PN CODE SEQUENCE FOR A DDS FREQUENCY HOPPING TRANSMITTER
}

\author{
M.M. Khodeir, K. Morsi, and A. Metkees \\ EE department, MTC University, Cairo, Egypt
}

\begin{abstract}
Frequency Hopping Spread Spectrum (FHSS) is a technique that wireless devices communicate such that the transmission frequencies are alternated in a pre-determined ordered hopping pattern. In tradition, the hopping sequence is controlled by using a pseudo-random code sequence (PN code) known only to both transmitter and receiver.

In this paper, the carrier frequency of a frequency shift keying FSK system is interfered by the surrounding environment. Thus, FHSS will be used instead to switch between multiple carriers within the available bandwidth to minimize the interference. Such a system requires that the generated PN code ensures that all carrier frequencies within the hopping pattern should be semiequally used during transmission. Several simulation scenarios were carried out to compare between a single long PN code and an accumulated short one. Finally, the developed FH system is implemented using an AD9910 Direct Digital Synthesizer (DDS) and an ALTERA Cyclone-III FPGA chip.

Results proved that, according to the system requirements, an accumulated short PN code offers noticeable residual correlations and provides a higher level of autocorrelation compared to a single long PN code. Results, also, verified that the developed FH system provides lower Bit Error Rate (BER) than the FSK one.
\end{abstract}

Keywords: Frequency Hopping Spread Spectrum, PN Code, DDS, FPGA. 


\section{Introduction}

Frequency hopping is the periodic changing of the carrier frequency of a transmitted signal. The sequence of carrier frequencies is called the frequency hopping pattern. The set of $\mathrm{M}$ possible carrier frequencies $\left[f_{1}, f_{2}, f_{3} \ldots \ldots \ldots, f_{M}\right]$ is called the hopset. The carrier frequency changes at a rate called the hop rate. Hopping occurs over a frequency band called the hopping band that includes $\mathrm{M}$ frequency channels. Each frequency channel is defined as a spectral region that includes a single carrier frequency of the hopset as its center frequency and has a bandwidth B large enough to include most of the power in a signal pulse with a specific carrier frequency. Figure (1) illustrates the frequency channels associated with a particular frequency-hopping pattern. The time interval between hops is called the hop interval. Its duration is called the hop duration and is denoted by $\mathbf{T}_{\mathbf{h}}$. The hopping band has bandwidth $\mathbf{W} \geq \mathbf{2 B}$. [2]

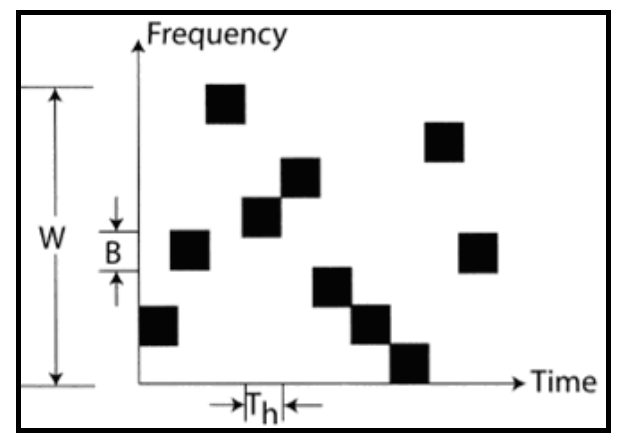

Fig.1. Frequency Hopping Patterns

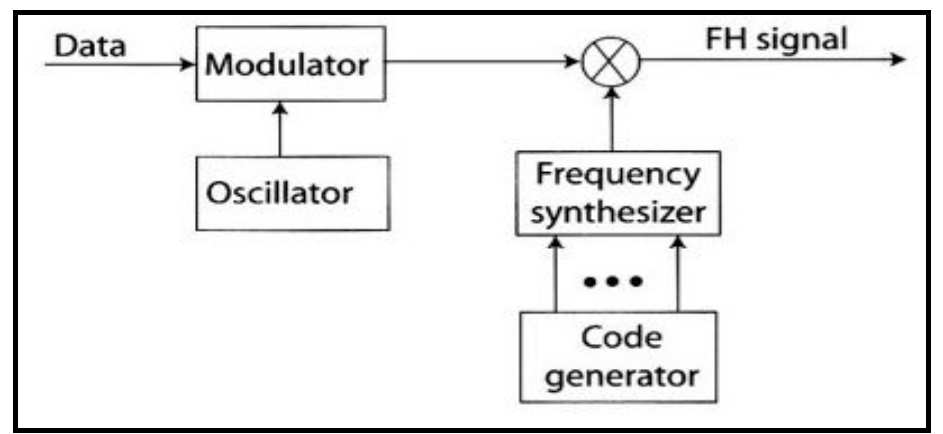

Fig.2. Frequency Hopping System

Figure (2) depicts the general form of a frequency-hopping system. The frequency synthesizers produce frequency-hopping patterns determined by the time-varying multilevel sequence specified by the output bits of the code generators. In the transmitter, the data-modulated signal is mixed with the synthesizer output pattern to produce the frequency-hopping signal. If the data modulation is some form of angle modulation $\varphi(t)$ then the received signal for the $\mathbf{i}^{\text {th }}$ hop is:

$$
\mathrm{S}(\mathrm{t})=\sqrt{2 S} \cos \left[2 \pi f_{i} \mathrm{t}+\varphi(t)+\varphi_{i}\right] \quad, \quad(i-1) T_{h} \leq t \leq i T_{h}
$$

Where $S$ is the average power, $f_{i}$ is the carrier frequency for this hop, and $\varphi_{i}$ is a random phase angle for the $i^{\text {th }}$ hop. The frequency-hopping pattern produced by the receiver synthesizer is synchronized with the pattern produced by the transmitter, but is offset by a fixed intermediate frequency, which may be zero. The mixing operation removes the frequency-hopping pattern from the received signal and, hence, is called de-hopping.

The remainder of this paper is organized as follows. Section 2 describes the pseudo-random code sequence (PN code). Direct Digital Synthesis (DDS) is explained in section 3. In Section 4, simulation results are presented and explained. Finally, the paper is concluded in Section 5.

\section{Pseudo-random code sequence (PN code)}

Pseudo-random code sequences (PN) are used as hopping pattern sources in frequency hopping systems. Each PN code sequence has its primitive polynomial and its initial state generated by a deterministic algorithm. The PN sequence generators are easy to construct using simple logic components (XOR gates and shift registers). The Linear generator polynomial $\mathrm{g}(\mathrm{x})$ of degree $\mathrm{m}>0$ is denoted by

$$
g(x)=g_{m} x^{m}+g_{m-1} x^{m-1}+\ldots \ldots . .+g_{1} x+g_{0}
$$


Where $\mathrm{g}(\mathrm{x})$ is a Primitive generating polynomial, $\mathrm{g}_{\mathrm{m}}$ and $\mathrm{g}_{0}=1$. and from the equation if the coefficient $\mathrm{g}$ is $=1$ it is mean that there is an (XOR) feedback at this shift register.

A linear code can be generated by a shift register with a configuration similar to the one shown in Fig.3. It is called Linear Feedback Shift Register (LFSR). [3]

- The number of flip flops, $n$, in the shift register

- The selection of feedback taps that are applied to one or more XOR gates

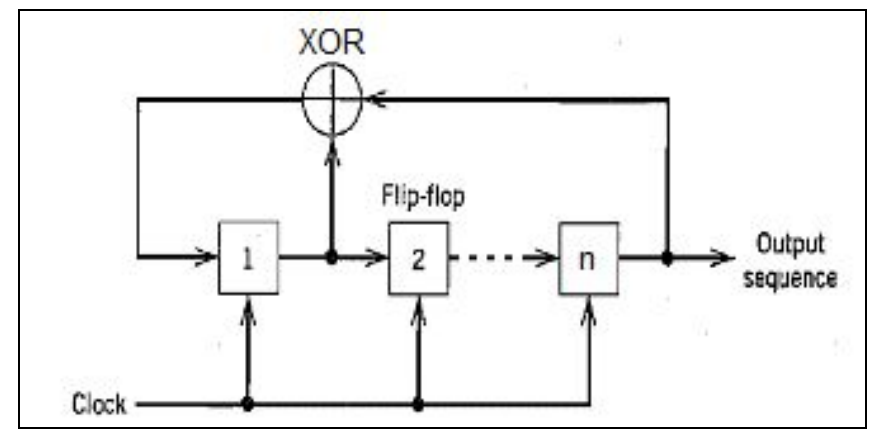

Fig.3. maximal sequence generator (LFSR)

Consider a code that is generated by a sequence generator that has $\mathrm{n}$ stages, then it would have a length of $\mathrm{N}=2^{\mathrm{n}}-1$ (a value of zero is not possible). The code is a "maximal sequence $(\mathrm{N})$ ") if the following properties apply to this code:

1- The number of ones in a sequence equals the number of zeros in the sequence plus one. Any m-sequence contains $2^{n-1} 1$ 's and $2^{n-1}-10$ 's

2- A Run Property is string of consecutive 1's or a string of consecutive 0's. In any msequence, one-half of the runs have length 1, one-quarter have length 2, one-eighth have length 3, and so on. In particular, there is one run of length $n$ of 1 's, one run of length n-1 of 0's.

3- The Autocorrelation property, the autocorrelation function of the m-sequence $(\mathrm{N})$ is periodic and binary valued, let binary symbols 0,1 of the sequence is denoted by $-1,+1$ respectively so that the autocorrelation function will be

$$
R(\tau)=\left\{\begin{aligned}
& 1, \tau=0, N, 2 N, \ldots . \\
&-\frac{1}{N}, \text { Otherwise }
\end{aligned}\right.
$$

The next table shows the primitive generator polynomial and the feedback connections (taps) which tabulated to be used to generate a maximal length of $\mathrm{PN}$ code sequence from $\mathrm{n}=(2-10)$

\begin{tabular}{|c|c|l|}
\hline $\mathbf{n}$ & $\mathbf{N = \mathbf { 2 } ^ { \mathbf { n - 1 } }}$ & \multicolumn{1}{|c|}{ Feedback Taps for m-sequences } \\
\hline 2 & 3 & {$[2,1]$} \\
\hline 3 & 7 & {$[3,1]$} \\
\hline 4 & 15 & {$[4,1]$} \\
\hline 5 & 31 & {$[5,3][5,4,3,2][5,4,2,1]$} \\
\hline 6 & 63 & {$[6,1][6,5,2,1][6,5,3,2]$} \\
\hline 7 & 127 & {$[7,1][7,3][7,3,2,1][7,4,3,2]$} \\
& & {$[7,6,4,2][7,6,3,1][7,6,5,2]$} \\
& & {$[7,6,5,4,2,1][7,5,4,3,2,1]$} \\
\hline 8 & 255 & {$[8,4,3,2][8,6,5,3][8,6,5,2]$} \\
& & {$[8,5,3,1][8,6,5,1][8,7,6,1]$} \\
& & {$[8,7,6,5,2,1][8,6,4,3,2,1]$} \\
\hline
\end{tabular}

\begin{tabular}{|c|c|l|}
\hline $\mathbf{n}$ & $\mathbf{N}=\mathbf{2}^{\mathbf{n}-\mathbf{1}}$ & \multicolumn{1}{|c|}{ Feedback Taps for $\mathbf{m}-$ sequences } \\
\hline 9 & 511 & {$[9,4][9,6,4,3][9,8,5,4][9,8,4,1]$} \\
& & {$[9,5,3,2][9,8,6,5][9,8,7,2]$} \\
& & {$[9,6,5,4,2,1][9,7,6,4,3,1]$} \\
& & {$[9,8,7,6,5,3]$} \\
\hline 10 & 1023 & {$[10,3][10,8,3,2][10,4,3,1][10,8,5,1]$} \\
& & {$[10,8,5,4][10,9,4,1][10,8,4,3]$} \\
& & {$[10,5,3,2][10,5,2,1][10,9,4,2]$} \\
& & {$[10,6,5,3,2,1][10,9,8,6,3,2]$} \\
& & {$[10,9,7,6,4,1][10,7,6,4,2,1]$} \\
& & {$[10,9,8,1,6,5,4,3][10,8,1,6,5,4,3,1]$} \\
\hline
\end{tabular}

Table 1.

The two important features that must be considered in the code are called cross-correlation and autocorrelation. Autocorrelation is a measure of similarity between the code and a phase-shifted 
version of itself. It can be defined as the number of times that the values of a phase shifted version of the code are equal to the values of the original code. This property is of extreme importance to our system. Another important property of the code is cross-correlation. This property is very similar to autocorrelation, but measures the amount of correlation of one sequence to a different sequence. This property is important to our system if it is to allow for CDMA.

\section{Direct Digital Synthesis (DDS)}

Direct digital synthesis (DDS) is a method of producing an analog waveform usually a sine wave by generating a time-varying signal in digital form and then performing a digital-to-analog conversion. Because operations within a DDS device are primarily digital, it can offer fast switching between output frequencies, fine frequency resolution, and operation over a broad spectrum of frequencies. With advances in design and process technology, today's DDS devices are very compact and draw little power. Fig.4 shows a DDS chip.

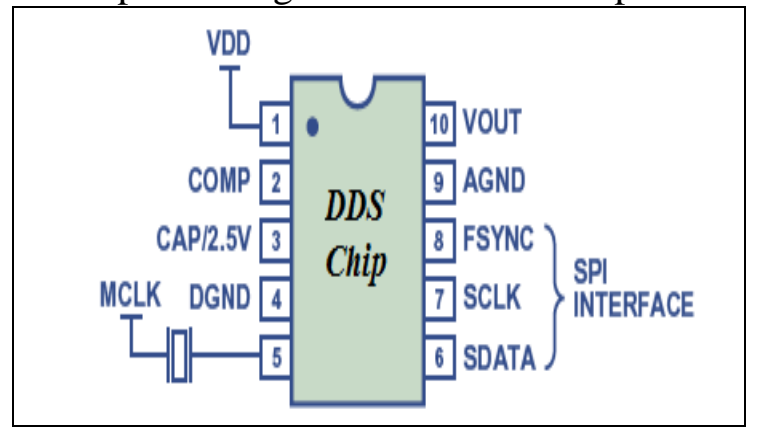

Fig.3.DDS chip

\subsection{The main benefits of using a DDS}

DDS are programmed through a high speed serial peripheral-interface (SPI), and need only an external clock to generate simple sine waves. DDS devices are now available that can generate frequencies from less than $1 \mathrm{~Hz}$ up to $400 \mathrm{MHz}$ (based on a 1-GHz clock). The benefits of their low power, low cost, and single small package, combined with their inherent excellent performance and the ability to digitally program (and re-program) the output waveform, make DDS devices an extremely attractive solution—preferable to less-flexible solutions comprising aggregations of discrete elements. The ability to accurately produce and control waveforms of various frequencies and profiles has become a key requirement common to a number of industries. Whether providing agile sources of low-phase-noise variable-frequencies with good spurious performance for communications, or simply generating a frequency stimulus in industrial or biomedical test equipment applications, convenience, compactness, and low cost are important design considerations. For example a DDS-based programmable waveform generator operating at $5.5 \mathrm{~V}$ with a $25-\mathrm{MHz}$ clock, consumes a maximum power of 30 milliwatt This relationship is found in the basic tuning equation for DDS architecture:

$$
f_{\text {ous }}=\frac{M \times f_{c}}{2^{n}}
$$

$f_{\text {out }}=$ output frequency of the DDS

$M=$ binary tuning word

$f_{c}=$ internal reference clock frequency (system clock)

$\mathrm{n}=$ length of the phase accumulator, in bits 


\section{Simulation}

The main problem of our system channel transmission is the Distortion of the signal in the way between the airborne system and the ground system due to (Noise - interference - jamming itc...). One solution to overcome this problem is to change the way of transmission. So that we operate the system with multiple carrier way (FH) using PSK modulation instead of single carrier with FSK modulation.

\subsection{The system requirements}

Table 2. Shows the system requirements and shows the calculation needed for the length of the PN code required for the system operation.

\begin{tabular}{l|l}
\hline \hline The whole test period & 3 minutes \\
\hline $\begin{array}{l}\text { The frame length } \\
\text { The Number of frames/sec is }=1 /\left(10 \times 10^{-3}\right)\end{array}$ & 10 msec. \\
\hline $\begin{array}{l}\text { The total number of frames in the whole test } \\
\quad(3 \text { min. } \times 60 \text { sec. } \times 100 \text { frame/ sec })\end{array}$ & 100 frame $/ \mathrm{sec}$. \\
\hline $\begin{array}{l}\text { In the system involved we use the Slow FH } \\
\text { each frame represented by (one Hop ) } \\
\text { the total hop numbers is = the number of frames }\end{array}$ & 18000 hopset \\
\hline $\begin{array}{l}\text { In the system involved we uses } 8 \text { carrier frequency } \\
\text { So we read the PN code by } \mathbf{3} \text { digits it means that each } 3 \\
\text { digit represent (one hop or one carrier ) } \\
\text { The PN code length required for the test is } \\
\geq 3 \times 18000\end{array}$ & $\geq 54000$ bit \\
\end{tabular}

First we generate the PN code using the LFSR circuit shown in Fig.6. and Fig.7. for the system, then according to the number of the frequency carriers we generate a LUT and read the PN code. In the system involved we read the PN code by 3-digit and compare with LUT and then selecting the frequency hop carrier will be used at this moment. Fig.4. shows the way of the frequency selection in the FHSS system.

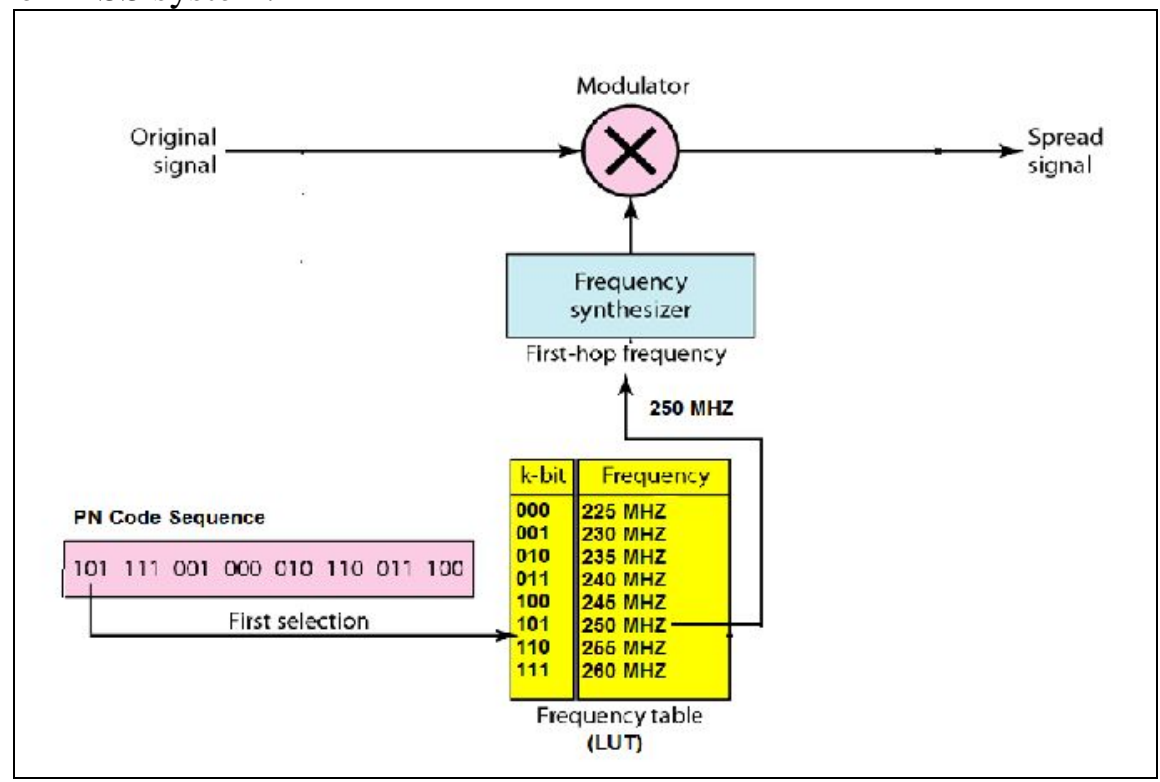

Fig.4.The frequency selection in FHSS system 


\subsection{Calculation of the $P N$ code length}

We have two ways for generating a PN code of length $\geq 54000$ bit we have to simulate and choose the best output result.

The $1^{\text {st }}$ way is: A generation of (long PN code sequence)

The $2^{\text {nd }}$ way is: A generation of (Accumulated short PN code sequence)

Fig.5. illustrates the two ways which will compare and select the optimal output PN code and optimal output hop-set.

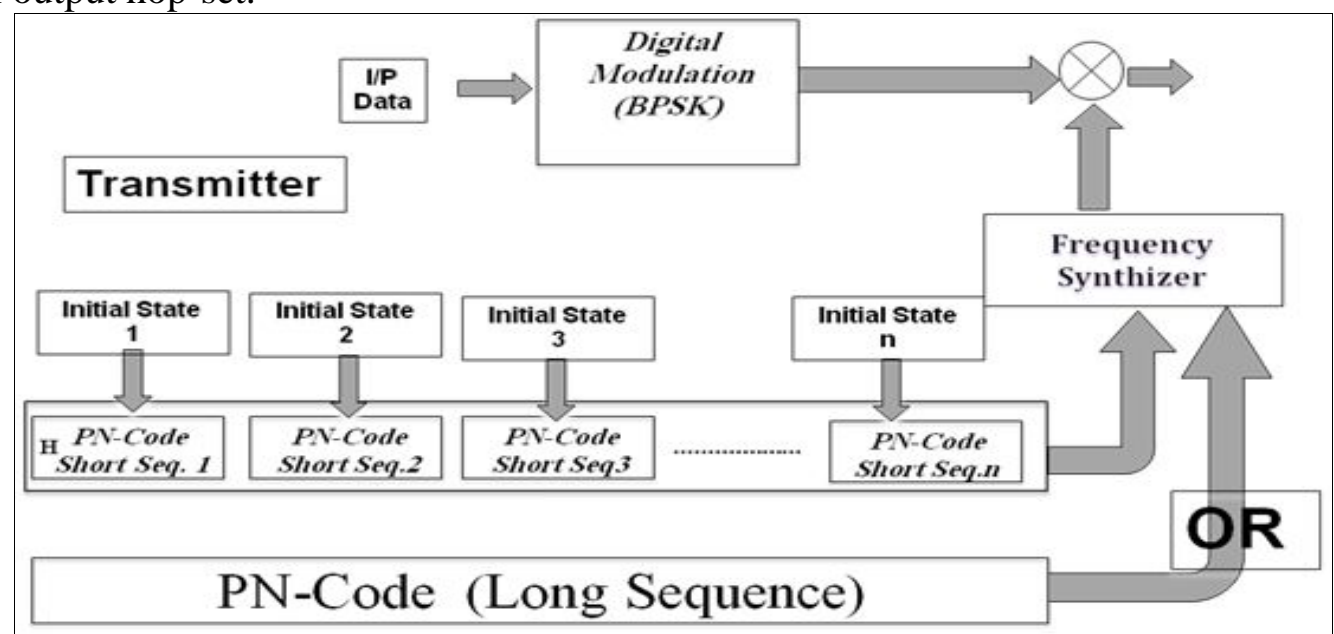

Fig.5. shows two ways to use PN code sequence in the hopping sequence

\subsubsection{A generation of (long $P N$ code sequence):}

$2^{\mathrm{n}}-1 \geq 54000$

$\mathrm{n} \ln 2=\ln (54000-1)$

$\mathrm{n} \geq 15.72$ so that $\mathrm{n}=16$

The PN code length $\mathrm{N}=2^{16}-1=65535$ bits.

So that we will use a generating polynomial of order (16) choosing it from the table 1 . of the max length of PN code sequence.

Fig.6. shows the circuit diagram of the (LFSR) circuit using for generation of the Long PN code It contents of (16) shift registers and (4) XOR logic gate for representing the 4-taps feedback.

$$
\mathbf{g}(\mathbf{x})=\left(X^{16}+X^{15}+X^{13}+X^{4}+1\right)
$$

\subsubsection{A generation of (Accumulated short $P N$ code sequence)}

Using a generating polynomial of order (8) and running the LFSR circuit 255 times (all available initial states) each time we use a different initial state .So that the total PN code length is $=\left(2^{8}-1\right) \times 255=255 \times 255=65025$ bits.

$$
\mathbf{g}(\mathbf{x})=\left(X^{8}+X^{4}+X^{3}+X^{2}+1\right)
$$

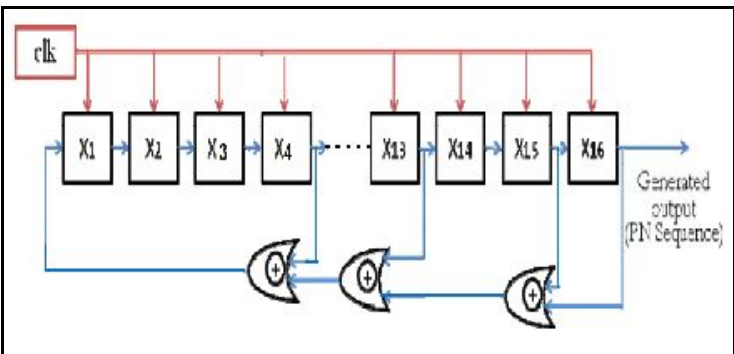

Fig.6. Circuit Diagram of 16- Bit LFSR with m- length Feedback Polynomial

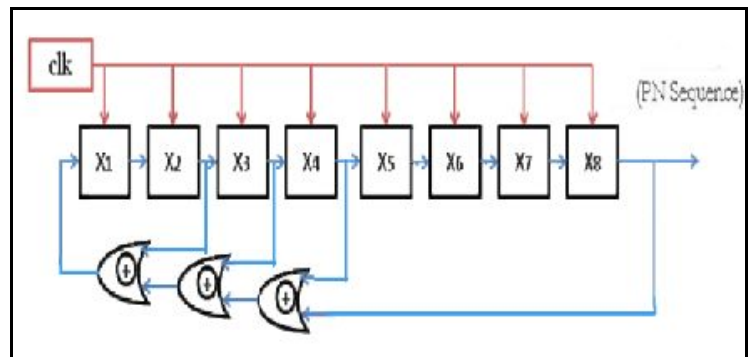

Fig.7. Circuit Diagram of 8-Bit LFSR with m-length Feedback Polynomial 
Table 3. Illustrates the comparison between the used parameters by the two ways

\begin{tabular}{c|c|c}
\hline & Long PN Code & $\begin{array}{c}\text { Accumulated Short PN } \\
\text { Code }\end{array}$ \\
\hline Polynomial g(x) Order Used & 16 & 8 \\
\hline The PN code Length & $\begin{array}{c}65535 \\
2^{16}-1\end{array}$ & $\begin{array}{c}65025 \\
255 \times 255\end{array}$ \\
\hline Polynomial g(x) Used & {$[16,15,13,4]$} & {$[8,4,3,2]$} \\
\hline Number of taps & $4-$ taps & {$[101110001]$} \\
\hline $\begin{array}{c}\text { Polynomial digits } \\
\text { representation }\end{array}$ & {$[11010000000010001]$} & {$[\mathbf{1 0 0 0 0 1 0 1}]$} \\
\hline Initial state & {$[0000000010000101]$} & 255 initial state \\
\hline Number of initial states & 1 initial state & 21675 \\
\hline Number of hops & 21845 & \\
\hline
\end{tabular}

\subsection{The Proposed Generating PN-Code}

We will compare between the histogram of the generated Hop Sequence from Both the Long sequence and the accumulated short-sequence. The target of this system is to generate a PN code sequence controls the hopping sequence semi-equally used during transmission to ensure that none of them possess a large hopped times which may become the affected one in that test environment.

Using the parameters calculated in the table 3. And writing a MATLAB m-file and simulate the tabulated data on both the two parameters (long PN code) and (short PN code). Fig. 8, 9 shows the simulation results of the histogram of both long and short code

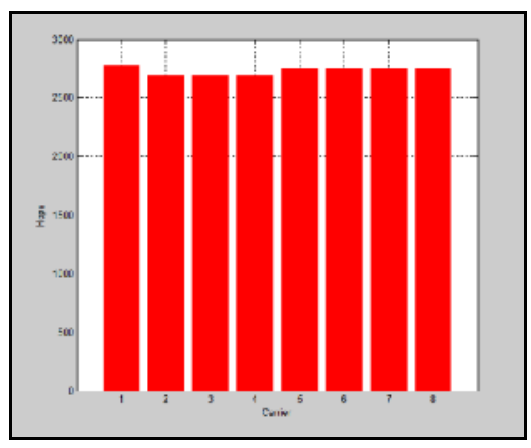

Fig.8. illustrates the output histogram for the (Long PN code sequence).

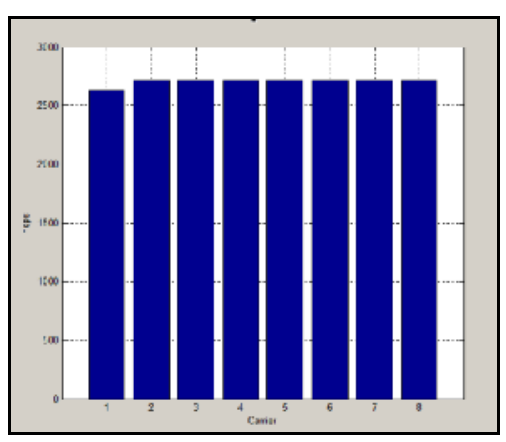

Fig.9. illustrates the output histogram for the (Accumulated short PN code sequence).

The histogram analysis conducted for the two generated hopping sequences, is depicted in Fig.8,9 From which it can be concluded that the Accumulated short PN code sequence selection mechanism can provide better uniform distribution of frequency channels over available band better than that provided by the Long one. 
The randomness of the (Accumulated short PN code sequence) and the (Long PN code sequence) can also be verified by the auto-correlation of each histogram. Fig.10 shows the auto-correlation properties of sort sequences and long sequences. So that we compare between the autocorrelation of the both histogram result so that the next figure shows the difference between the both autocorrelation.

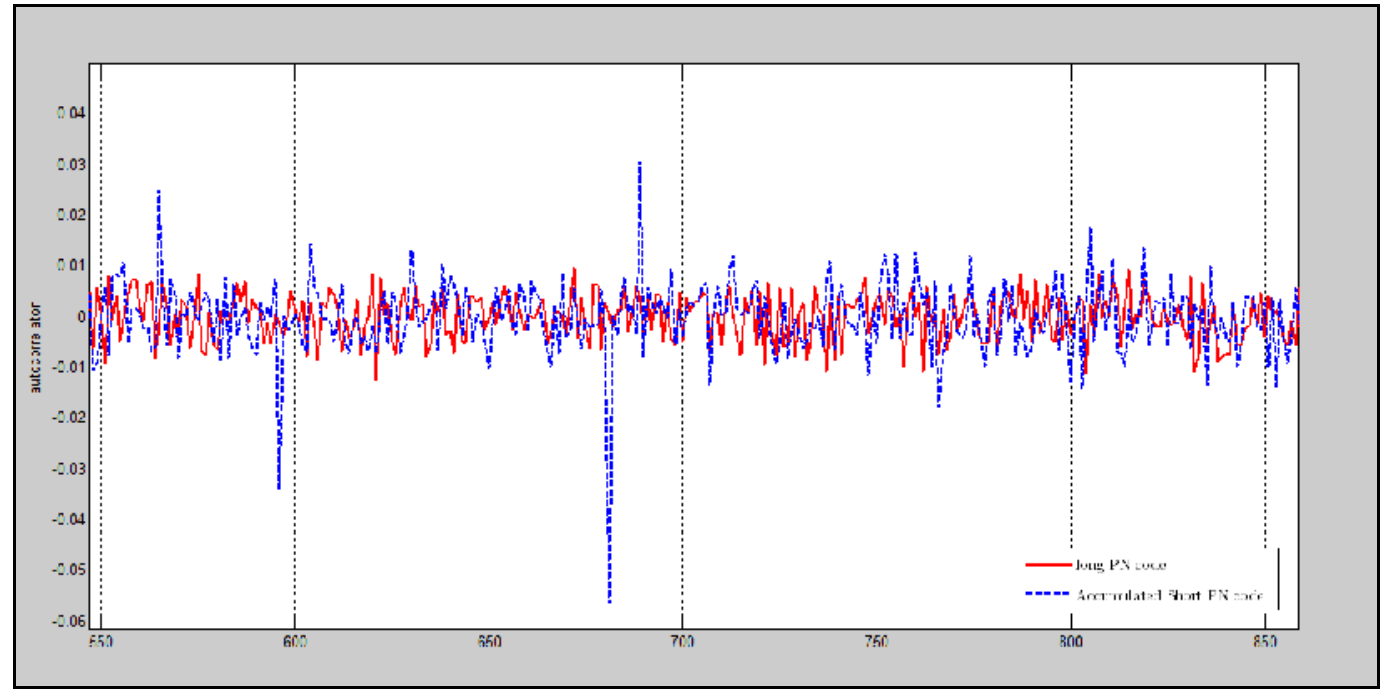

Fig.10.shows the autocorrelation between Long and Acc. Short PN code

On the other hand, the FHSS system will use the BPSK digital modulation instead of the FSK modulation due to the better BER probability over the $\mathrm{E}_{\mathrm{b}} / \mathrm{N}_{0}$ related to Fig.11

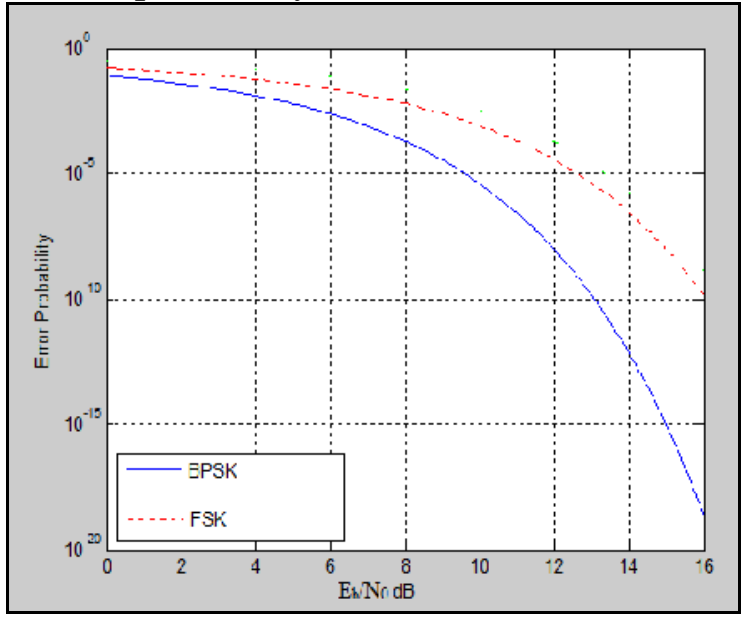

Fig.11.relation between BER of both BFSK and FSK modulation

Carrying out the simulation of obtaining the histogram and the output autocorrelation of another two systems having a PN code length of 4095 and 16383 using caparison between $\mathrm{g}(\mathrm{x})$ of order $(6,12)$ in the 1 st system and between $\mathrm{g}(\mathrm{x})$ of order $(7,14)$ of the 2 nd system.

Fig.12, 13, 14,15,16,17 shows the histogram and autocorrelation of the both system which related to the same conclusion that the Accumulated Short PN code system is much better than the Long PN code. 


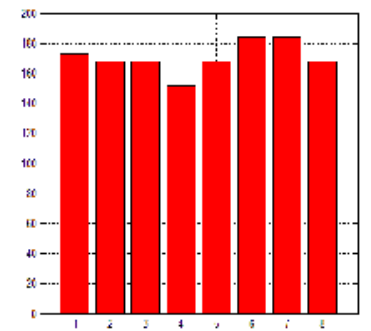

Fig.12. histogram of the $1^{\text {st }}$ long PN code seq.

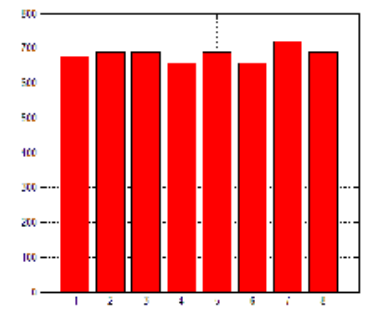

Fig.15. histogram of the $2^{\text {nd }}$ long PN code seq.

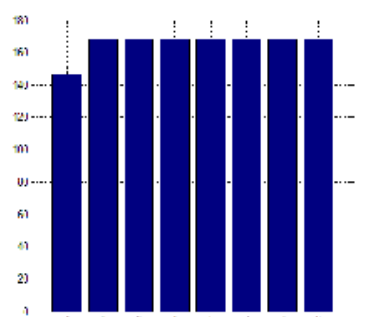

Fig.13. histogram of the $1^{\text {st }}$ short PN code seq.

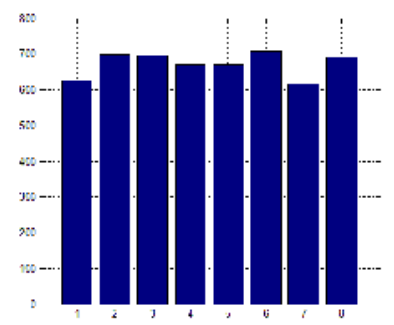

Fig.16. histogram of the $2^{\text {nd }}$ short PN code seq.

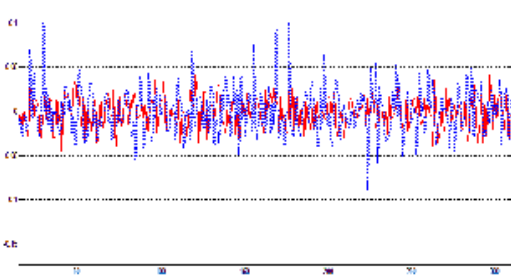

Fig.13.output autocorrelation of the $1^{\text {st }}$ long and short PN code seq.

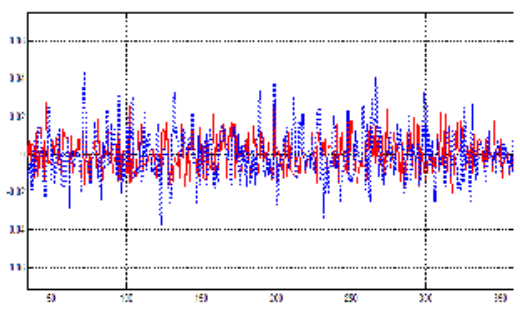

Fig.17.output autocorrelation of the $2^{\text {nd }}$ long and short $P N$ code seq.

\section{Hardware implementation}

The old system is using single carrier with FSK modulation so we upgrade the system to be FHSS using PSK modulation, trying to eliminate the problems affected by the environment, in addition the BER of the PSK is much better than the FSK as shown in Fig.11

It is one of the ways trying to improve the old system

\subsection{Synchronization of the frame with the DDS}

In the System involves we use the slow frequency hopping technique its mean that every transmitted frame will transmitted via independent hop carrier, moreover upgrading the system needs a synchronization between the transmitted frames with output hop carrier. The system requires an indication to the DDS chip to synch between the frame and the output hop carrier. The usage of FPGA kit (shown in Fig.12) and designing VHDL code give an output pulse indication to the DDS to control the output carrier for each input frame to the system.

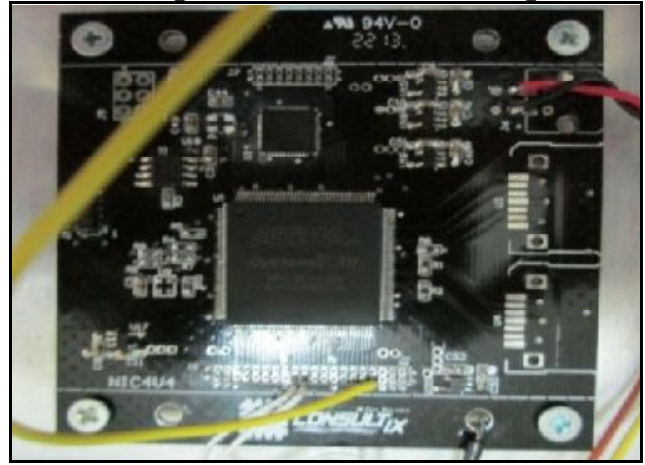

Fig.18. ALTERA FPGA kit

Here is the ALTERA FPGA kit used for implementation of the additional hardware for synchronization the frame with the output hop carrier 
The next figure shows the internal design of the external FPGA kit designed for frame synchronization with the DDS chip.

\begin{tabular}{|c|c|c|c|c|}
\hline clock $;$ & & Poul $<19.0>$ & ex<19.0> AND gate pulse & Pulse out \\
\hline enable, & & $\begin{array}{l}\text { Serial to Paralell } \\
\text { Converter }\end{array}$ & & Output Pulse \\
\hline Sin in & Sin & & & \\
\hline
\end{tabular}

Fig.19.The internal design schematic of the FPGA kit

The frame data input is serial data and then converted to a parallel sequence, ANDing the frame data simultaneously with the stored frame header in the FPGA kit memory. Results in an output pulse will enter the DDS to switch the hop carrier order.

Fig.14 shows the simulation output using the ISE tool kit for the output pulse signal

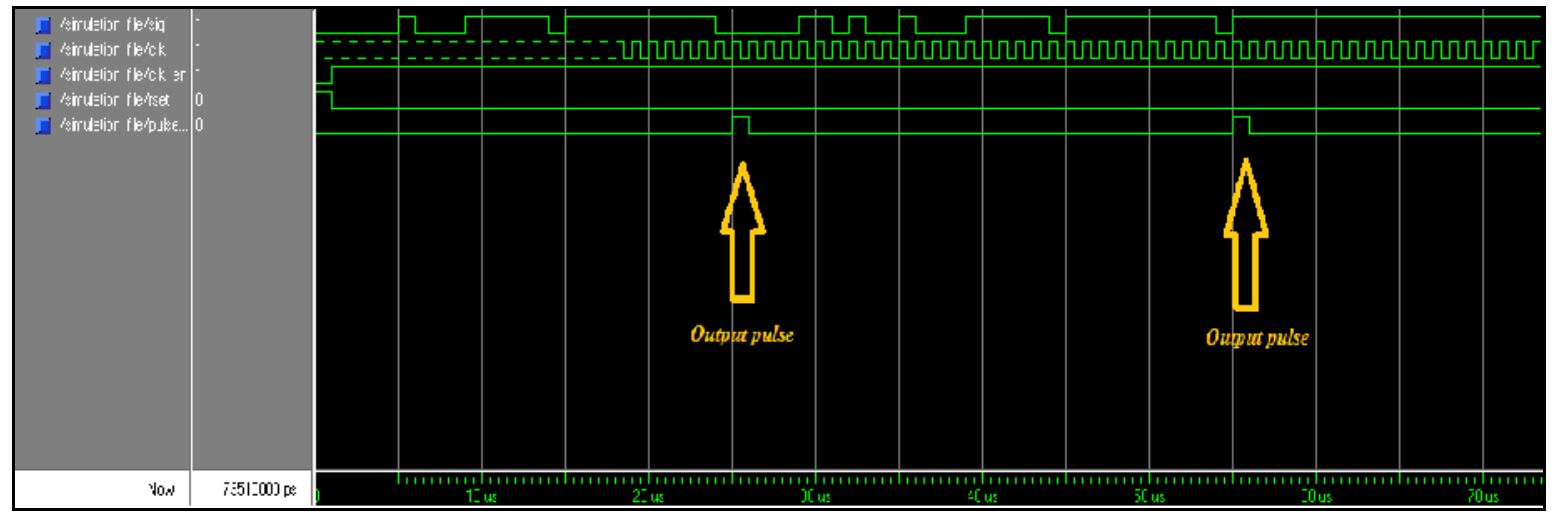

Fig.20.The VHDL simulation output of the pulse using for synchronization

\subsection{Hardware Implementation Setup and Outputs}

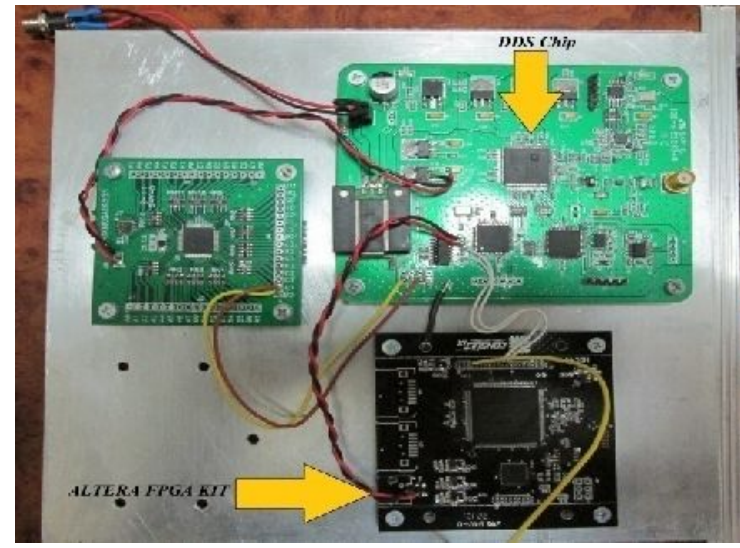

(a)

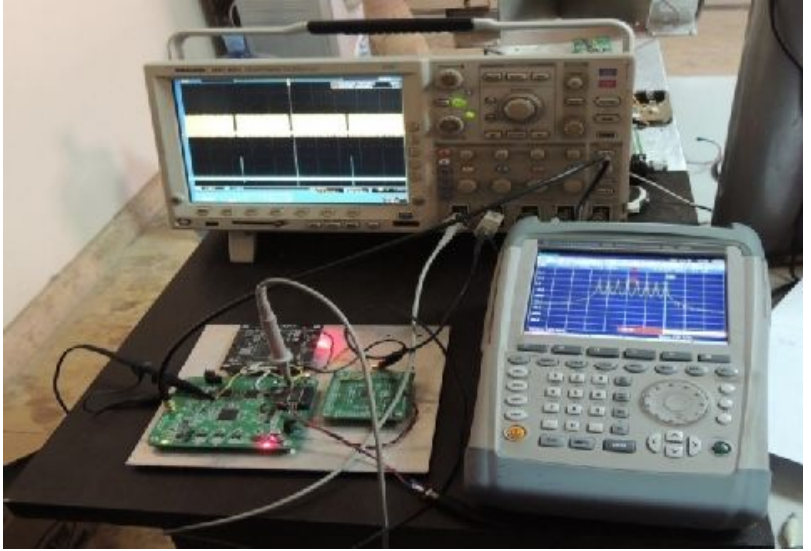

(b)

Fig.21.a , Fig.21.b shows the instrumentation set up for the hardware 


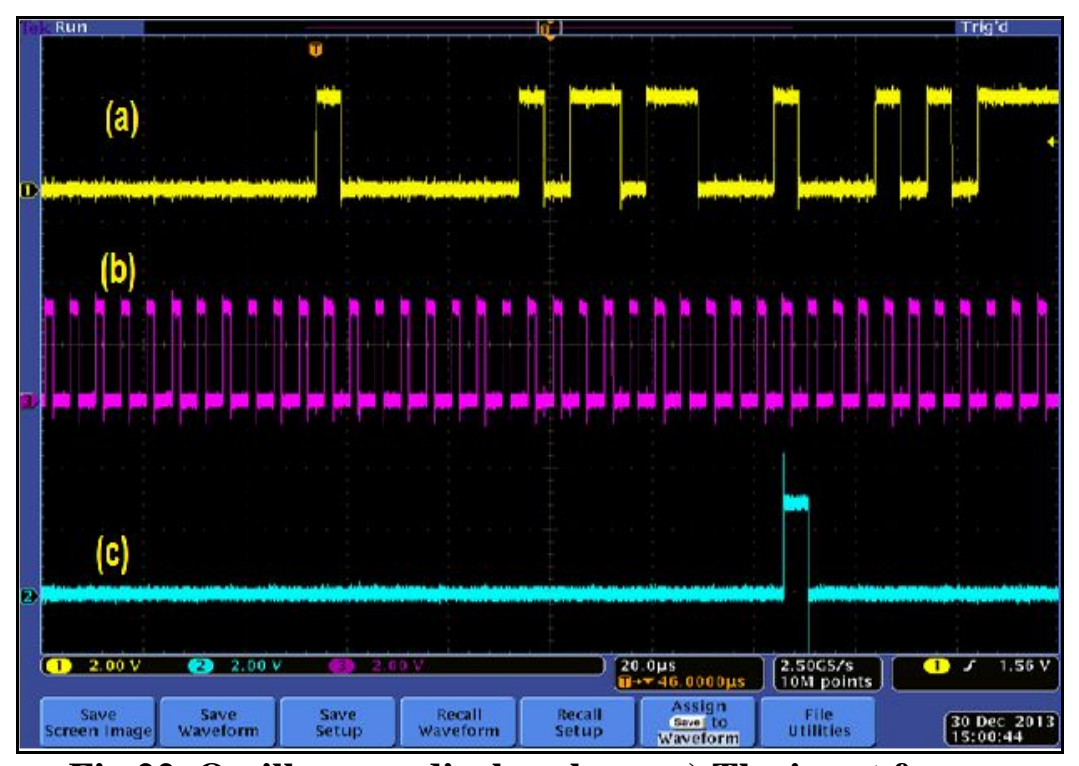

Fig.22. Oscilloscope display shows a) The input frame b) The input clock. c) The output pulse

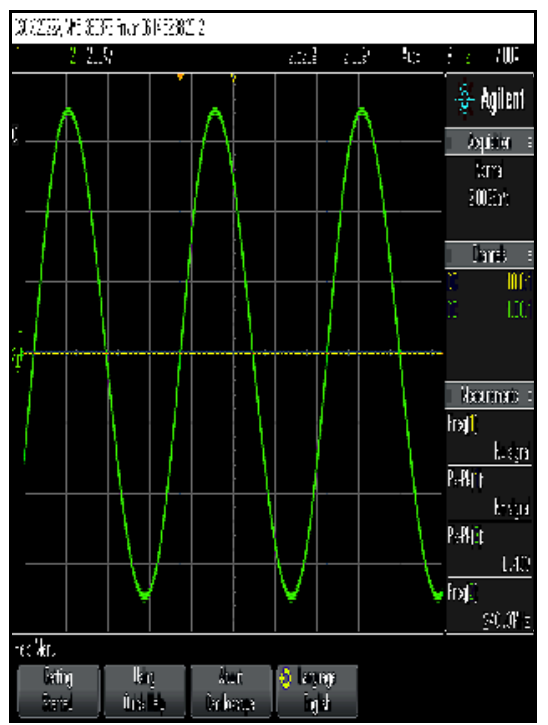

Fig.23. shows the DDS output frequency at $240 \mathrm{MHZ}$

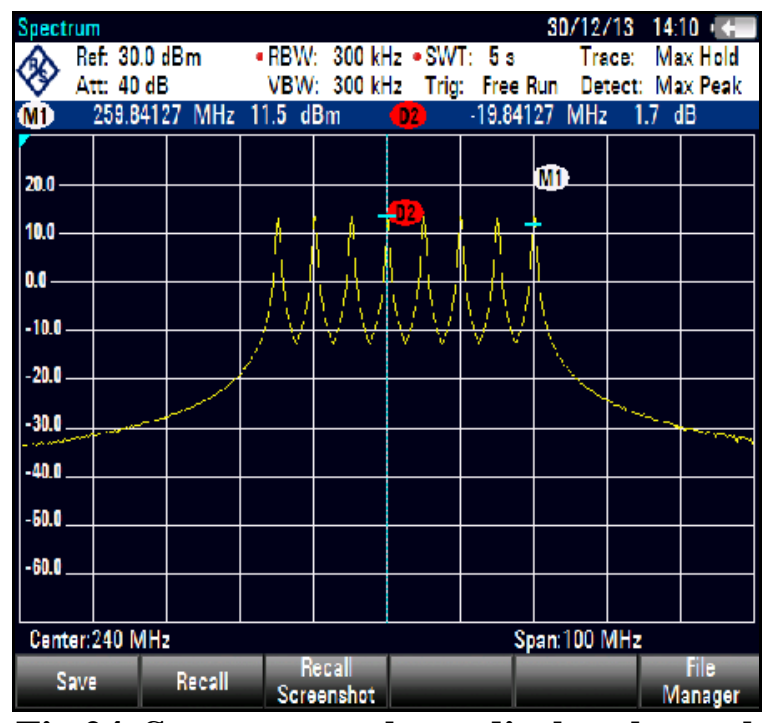

Fig.24. Spectrum analyzer display shows the output 8 hop carriers frequencies

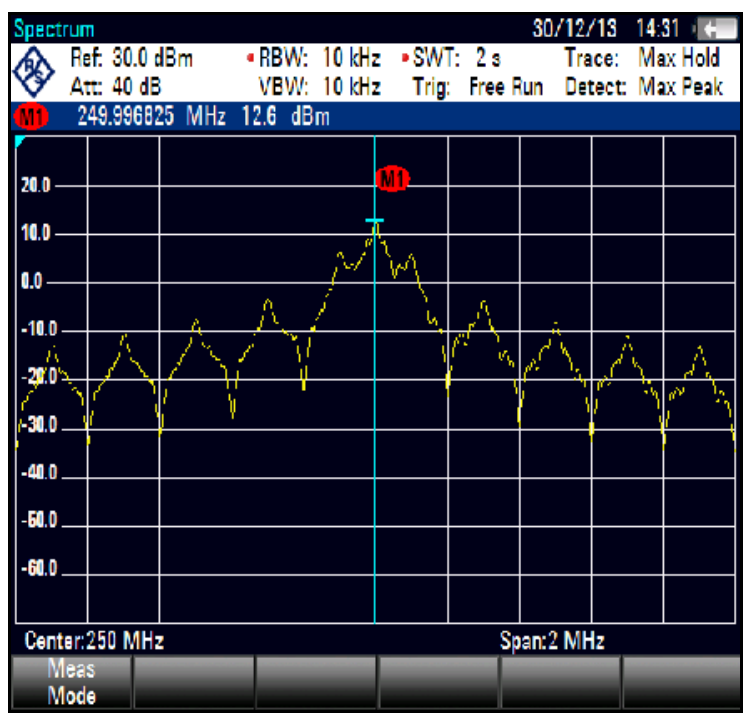

Fig.25. Spectrum analyzer display shows the output hop carrier frequency at $250 \mathrm{MHZ}$ 


\section{Conclusion}

From the system involved we conclude that the usage of (Accumulated Short PN Code sequence) is much better that using a (Long PN Code sequence) in the generation of the hopping sequence for achieving frequency hopping sequence pattern of carriers should be semi-equally used during transmission. Moreover the output of the histogram and the results of the autocorrelation of each code show the best PN code.

\section{References}

[1] D. Torrieri. Principles of Spread-Spectrum Communication Systems. Springer, $2^{\text {nd }}$.ed., 2011.

[2] R. M. Buehrer. Code Division Multiple Access (CDMA). Morgan \& Claypool Publishers, 2006.

[3] Ding Jun, Li Na, Guo Yixiong, "A high-performance pseudo-random number generator based on FPGA" 2009 International Conference on Wireless Networks and Information Systems.

[4] R. L. Pickholtz, D. L. Shilling, L. B. Milstein. Theory of Spread-Spectrum Communications - A Tutorial. In IEEE Transactions on Communications, 1982.

[5] Simon Haykin, Communication Systems, $4^{\text {th }}$.ed., Wiley, New York, 2001.

[6] Jiang Hao, Li Zheying, "On the Production of Pseudo-random Numbers in Cryptography" in Journal Of Changzhou Teachers College of Technology, Vo1. 7 , No. 4, Dec. 2001. 